samples. Between 10/18 - 8/20 6,401 reports received a consult. The data contained herein are derived from those consults. Consultants record information on prior meds, reason for failure or intolerability, potential risk-associated or useful drugs based on the genetic variants. Consultants only recommend specific drugs and doses consistent with a published PGx guideline.

Results. The 5 most commonly discussed genes were SLC6A4, MTHFR, CACNA1C, COMT and BDNF. The 3 most commonly discussed drugs were fluoxetine, lithium and duloxetine. The most common reasons for drug failure were inefficacy and drug induced "agitation, irritability and/or anxiety". SSRIs were the most common class of discontinued drug; sertraline, escitalopram and fluoxetine were the three most commonly reported discontinuations and were also the 3 most likely to be associated with "no improvement". Aripiprazole was the most commonly reported discontinued atypical antipsychotic. The providers rated $94 \%$ of consultations as extremely or very helpful at the time of consult. An independent validation survey of 128 providers confirmed these ratings, with $96 \%$ reporting a rating of "very helpful" or "extremely helpful". In addition, 94\% reported that these consults were superior to PGx consults provided through other laboratories. Patient characteristics captured during consults via a Clinical Global Impressions-Severity (CGI-S) scale revealed that the majority of patients were moderately (54\%) or markedly ill (23\%). The most frequent symptoms reported were depression, anxiety, insomnia and inattentiveness.

Discussion. The large variety of psychotropic drugs available to providers, and their highly variable response rates, tolerability, capacity for drug-drug interactions and metabolic pathways present a challenge for even expert psychopharmacologists. Consultation with experts in PGx provides additional useful information that may improve outcomes and decrease healthcare resource utilization. This database may provide future opportunities for machine learning algorithms to further inform implications of included gene variants.

Funding. Genomind, Inc.

\section{A Longitudinal Study to Assess Antidepressant Treatment Patterns and Outcomes in Individuals with Depression in the General Population}

\author{
Maurice M. Ohayon, MD, DSc, $\mathrm{PhD}^{1}$, \\ Maggie McCue, MS, $\mathrm{RD}^{2}$, Andrew Krystal, MD, $\mathrm{MS}^{3}$, \\ Lambros Chrones, MD², Maelys Touya, PharmD, MSc ${ }^{4}$, \\ Debra Lawrence, MS, MBA, PhD², \\ Sheetal Patel, MD, $\mathrm{MS}^{4}$ and Marie Lise Cote, $\mathrm{MA}^{5}$
}

${ }^{1}$ Stanford University School of Medicine, Sleep Epidemiology Research Center (SSERC), Division of Public Mental Health and Population Sciences, Palo Alto, CA,
USA, ${ }^{2}$ Takeda Pharmaceuticals Inc., Lexington, MA, USA, ${ }^{3}$ University of California San Francisco, Weill Institute for Neurosciences, Departments of Psychiatry and Neurology, San Francisco, CA, USA, ${ }^{4}$ Lundbeck LLC, Deerfield, IL, USA, and ${ }^{5}$ Centre d'évaluation et de Statistique (CES), Montreal, Canada

Presenting Author: Maurice M. Ohayon

\begin{abstract}
Study Objectives. Depression is an important cause of disability in the United States (US). The care experience of major depressive disorder (MDD) is highly variable and has only been documented to a limited degree. This study examines the prevalence incidence and treatment patterns for MDD in the US general population.

Methods. In this longitudinal study 2 interview waves were conducted between 2002 and 2015. The initial wave (W1) was carried out with 12,218 individuals from the general population in 8 US states with participants aged 18 years or older. In the second wave (W2) 10,931 of the initial participants agreed to be interviewed again 3 years later; the analyses were carried out for individuals who participated in both interviews $(\mathrm{N}=10,931)$. Diagnosis of MDD was confirmed according to Diagnostic and Statistical Manual of Mental Disorders Fifth Edition (DSM-5) criteria.
\end{abstract}

Results. The 3-year incidence of MDD was 3.4\% (95\% CI 3.1\%$3.7 \%$ ). The prevalence of MDD was 5.1\% (95\% CI 4.7\%-5.5\%) and $4.2 \%$ (95\% CI 3.8\%-4.6\%) in W1 and W2, respectively. The percentages of participants who achieved partial and complete remission were $4.4 \%$ (95\% CI $4.0 \%-4.8 \%)$ and $3.9 \%(95 \% \mathrm{CI}$ $3.5 \%-4.3 \%)$ in W1 compared with $7.9 \%$ (95\% CI 7.4\%-8.4\%) and $4.4 \%$ (95\% CI $4.0 \%-4.8 \%)$ in $\mathrm{W} 2$, respectively. The prevalence of MDD was $13.4 \%$ and $16.5 \%$ in W1 and W2, respectively, when including participants with MDD partial and complete remission episodes. $61.9 \%$ of participants with an MDD diagnosis in W1 had at least one associated comorbidity. $41.8 \%$ of participants with an MDD diagnosis at W1 still reported significant depressive symptoms at W2. 19.9\% of participants in partial remission and $5.5 \%$ of participants in complete remission in W1 did not achieve remission in W2. 52.2\% and $42.9 \%$ of participants with MDD were treated with an antidepressant (AD) in W1 and W2, respectively; selective serotonin reuptake inhibitors (SSRIs) were the most commonly prescribed $(34.7 \%$ in $\mathrm{W} 1$ vs $28.3 \%$ in $\mathrm{W} 2$ ). ADs were mainly prescribed by primary care physicians (45.7\%) followed by psychiatrists (31.4\%), neurologists $(2.5 \%)$, and other specialties $(7.9 \%)$. The average duration of treatment was 36.9 (SE 2.4) months. More than one-third of AD users in $\mathrm{W} 1$ expressed dissatisfaction with their $\mathrm{AD}$ treatment which translated into changes in types of antidepressant in W2. Conclusion. Depression affects a sizable part of the general population in the US with a prevalence of MDD at $13.4 \%$ $16.5 \%$; yet MDD remains largely undertreated as shown by the finding that only about half (52\%) of individuals in this study who met the diagnostic criteria for MDD were treated with an antidepressant (SSRI being the most common treatment). In addition, more than a quarter of patients with MDD in this study did not achieve remission after initial treatment underscoring the challenges in successful antidepressant treatment of MDD.

Funding. Takeda Pharmaceuticals U.S.A. Inc. and Lundbeck LLC 